\title{
Deep learning approaches in predicting ADMET properties
}

\author{
Elena L Cáceres ${ }^{1,2}$ (D), Matthew Tudor ${ }^{3}$ \& Alan C Cheng*,1 (iD) \\ ${ }^{1}$ Computational \& Structural Chemistry, Merck \& Co., Inc., South San Francisco, CA 94080, USA \\ ${ }^{2}$ Department of Pharmaceutical Chemistry; Graduate Program in Biological \& Medical Informatics; Department of Bioengineering \\ \& Therapeutic Sciences; Bakar Computational Health Sciences Institute; Kavli Institute for Fundamental Neuroscience; Institute for \\ Neurodegenerative Diseases, University of California San Francisco, San Francisco, CA 94143, USA \\ ${ }^{3}$ Computational \& Structural Chemistry, Merck \& Co., Inc., West Point, PA 19486, USA \\ *Author for correspondence: alan.cheng@merck.com \\ "The results suggest that learned representations encoded by GCNNs may be better at extracting \\ structure-activity relationships from smaller molecules that extrapolate in chemical space to \\ larger molecules."
}

First draft submitted: 3 August 2020; Accepted for publication: 19 August 2020; Published online: 30 October 2020

Keywords: ADME/Tox • computational chemistry and molecular modelling • drug design • drug discovery $\bullet$ drug metabolism

Since the early days of Lipinski's rule of five, the field of predictive ADMET (absorption, distribution, metabolism, excretion and toxicity) in medicinal chemistry has expanded in importance and has grown to include areas such as high-throughput assay development, data mining, data visualization, machine learning and structure-based modeling [1,2]. Many studies have demonstrated the role of effective application of in silico predictive ADMET models in accelerating the identification of small molecules with improved efficacy, safety and dose [1].

Machine learning models are now routinely used by drug discovery teams to predict properties of small molecules based off their chemical structure. The first methods were 'simple' linear models such as those used in Free-Wilson and Hammett analyses and these are still in use today due to their easy interpretability and effectiveness on small datasets [2]. Nonlinear models were later established to capture more complex relationships between structure and activity, and such approaches include support vector machines, recursive partitioning methods (such as random forest, Cubist and XGBoost) and deep learning methods such as deep artificial neural networks (DNNs) [2,3].

Deep learning-based approaches are showing increasing promise and usefulness for ADMET prediction, fueled by increasing computational power, larger datasets generated in a standardized manner, and adaptation of image and language processing advances to chemistry [1,2]. Here, we first explore the role of deep learning in recent ADMET prediction performance advances and then discuss ongoing work to address challenges in evaluating, interpreting and implementing deep learning for molecular design.

\section{Rise of deep learning for ADMET prediction}

A 2012 Kaggle competition sponsored by Merck \& Co., Inc., (NJ, USA) generated excitement around DNNs for ADMET prediction when researchers reported that simple DNNs yielded a $10 \%$ mean improvement in $\mathrm{R}^{2}$ performance across 15 large assay datasets when compared with widely-used, prevailing random forest (RF) models [4]. $\mathrm{R}^{2}$ is the squared Pearson correlation coefficient that ranges from $0-1$ (higher is better) and measures how well the prediction matches the experimental data, usually on a left-out data subset [4]. Other researchers, including those at Vertex Inc., (MA, USA) [5], Eli Lilly \& Co. (IN, USA) [6] and Bayer AG (Berlin, Germany) [7], similarly found that simple DNNs (in these cases, fully-connected DNNs) were comparable or slightly improved over prevailing models when trained on large, proprietary ADMET datasets. Despite promising results, many practitioners at the time felt that the impact of the generally modest performance improvement seen in these early DNNs did not justify the major increase in resources, computational and human, required to maintain such models. 
The winning Kaggle competitor demonstrated dramatic performance improvements on some Kaggle end points using a multitask DNN that simultaneously learned all assay tasks within a single model [4,7-9]. However, thoughtful investigation into these DNNs led to a more nuanced understanding of their performance for ADMET prediction. $\mathrm{Xu}$ et al. and Kearnes et al. raised concerns that multitask models derived a large part of their improved performance from 'memorizing' or 'leaking' molecules regardless of the relevance of assisting assays [2,5,7,9]. This behavior manifests advantageously when molecules are structurally similar and assays are related, but potentially worsens or does not improve predictions when molecules are similar and assays are unrelated.

In an interesting demonstration of this, Wenzel et al. trained a multitask fully-connected DNN using large Sanofi-Aventis AG liver microsome stability datasets ( 50,000 compounds) for three species and found predictive performance improved for human liver microsomes (HLM), but worsened slightly or stayed the same for rat and mouse liver microsomes (RLM and MLM, respectively) [8]. However, when overlapping molecules were disallowed between the prediction molecules for HLM and any training molecules (regardless of species), essentially no benefit was seen from multitask training. Upon addition of five additional species with small liver microsome datasets of 200-1500 compounds each to create an eight-species multitask model, Wenzel et al. saw mixed predictive performance, with macaque predictions benefiting ( $\mathrm{R}^{2}$ increased by 0.09 or $\left.15 \%\right)$, but monkey predictions worsening $\left(\mathrm{R}^{2}\right.$ decreased by 0.09 or $\left.24 \%\right)$. Montanari et al. explored the benefits of multitask fully-connected DNNs and concluded it was 'trial-and-error type of work' [7].

\section{Learned featurization improves predictive performance}

As enthusiasm tempered for multitask DNNs, a new twist on deep learning was emerging. Both the prevailing models (such as RF models) and the DNN models described above require a static 'fingerprint' representation of each compound. Fingerprints are vectors of discrete numbers where each number represents the presence (zero or one) or count of a chemical fragment [2]. Duvenaud et al. proposed that instead of enumerating possible fragments into a static fingerprint, a graph convolutional $\mathrm{DNN}(\mathrm{GCNN})$ approach could be used to dynamically learn a fingerprint optimized for the most relevant chemical information, where adjacency in the vector representation encodes fragment similarity relevant to an assay end point of interest [2,5,10-14].

In a recent study, Feinberg et al. investigated prediction of 31 ADMET end points using a state-of-the-art GCNN and large, standardized assay datasets at Merck \& Co., Inc. [14]. Their single-task GCNN showed strong performance improvements over RF for about a third of end points, with improvements in $\mathrm{R}^{2}$ of $0.15-0.31$ or $45 \%-133 \%$. These end points include plasma protein binding (PPB), HLM and RLM, cytochrome p450 3A4 (CYP3A4) and 2D6 inhibition, hERG binding and kinetic solubility in water. Especially remarkable was the large improvement for rat and human PPB ( $\mathrm{R}^{2}$ improvements of 0.31 and 0.19 , respectively). Another third of end points showed smaller improvements $\left(\mathrm{R}^{2}\right.$ improvements of $\left.0.05-0.14\right)$ that may be meaningful. Conversely, a third of end points showed minimal or no improvement, notably including all in vivo pharmacokinetic end points. The authors saw additional performance improvements by adding a multitask approach for featurization and this improvement was most notable for hepatocyte stability, permeability and P-glycoprotein efflux. Overall, they found that their multitask GCNNs performed equivalently or better than single-task GCNNs, which, in turn, performed equivalently or better than RF models.

Using a different state-of-the-art GCNN, Yang et al. showed notable performance relative to RF on smaller proprietary industrial ADMET datasets as well as on public datasets. On four large Amgen Inc., (CA, USA), datasets (rat PPB, solubility, RLM and human PXR activation), their ensemble single-task GCNN models showed median improvement of $14 \%$ in root-mean-square error compared with RF models using fingerprints [11]. Liu et al. compared yet another single-task GCNN model approach to a Cubist approach using five different Amgen Inc. datasets [15], and found that single and multitask GCNNs showed $10 \%$ and $38 \%$ mean improvements in $\mathrm{R}^{2}$ performance, respectively, over Cubist models. Liu et al. found that single-task GCNNs consistently outperformed Cubist in HLM, CYP3A4 and solubility end points, while both performed equivalently for human PXR activation prediction. They applied a multitask GCNN to modeling of multiple solubility and PXR subsets and these models consistently outperformed Cubist models. These studies suggest that GCNNs are not only competitive to RF and Cubist models, but in some cases yield robust increases in predictivity.

Another study by Montanari $e$ e al. used eight Bayer AG proprietary ADMET datasets with 39,000-236,000 compounds each to compare the learned representations from GCNNs against two fingerprint-based approaches, simple fully-connected DNNs and RFs [7]. Multitask GCNNs improved predictive performance the most over prevailing RF models. With membrane affinity and PPB end points, they saw $\mathrm{R}^{2}$ improvements of $0.26-0.28$ or 
65-67\%. Overall, they found that deep learning approaches out-performed RF, multitask models were competitive or outperformed single-task models, and GCNNs outperformed fully-connected DNNs of the same singletask or multitask training type.

Taken together, recent work suggests models built using GCNNs can consistently deliver better or equivalent predictive performance compared with prevailing approaches (such as RF, Cubist and support vector machine), and, in some cases, demonstrate impressive gains. We highlight PPB and RLM/HLM end points as two end points that see strong improvement in multiple studies, especially with larger industrial datasets ( $>15,000$ compounds). Multitask GCNNs appear to slightly outperform single-task GCNNs, although ensemble single-task GCNNs reported by Yang et al. [11] are putting up a solid fight for comparative performance (manuscript in preparation).

\section{Measuring how models generalize for medicinal chemistry}

The performance metrics reported in the earlier sections use datasets split into a learning subset and a testing subset using either a time-based split or a scaffold-based split [16]. These two splits are preferred over a random split, which is considered less reflective of real-world drug discovery settings and gives an unrealistically optimistic assessment of prospective performance. In time splits, earlier data is used for training, and more recent data for testing. This simulates the drug discovery setting where researchers relentlessly evolve molecules into novel chemical space over time. Recent work suggests that a scaffold split - which assigns different compound clusters or scaffolds to the training and testing sets - can be useful when temporal splits are insufficiently diverse, where there is no testing date available or where there are concerns over model generalizability. Using industrial datasets, scientists at our laboratories found models perform better on time splits than on scaffold splits of the same datasets, and both are clearly more challenging than random splits $[9,16]$. On the other hand, studies using Amgen Inc. (CA, USA), Novartis AG, (Basel, Switzerland), and Bayer AG (Berlin, Germany), datasets found scaffold splits to be slightly more difficult than time splits [11]. Our takeaway is that scaffold split is a good, but imperfect, estimate of time split and both are clearly more predictive of expected performance in medicinal chemistry progression than random split.

To assess the potential of GCNN models for chemical space extrapolation, Feinberg et al. recently proposed a time plus molecular weight (MW) split [14]. In this split, models are trained on earlier compounds with MW $\leq 500 \mathrm{Da}$ and tested on later molecules with $\mathrm{MW} \geq 600 \mathrm{Da}$. Under this split, GCNNs were able to maintain much of their performance on the third of assays where they performed strongly [14]. This contrasts with the poor performance of RF models. The results suggest that learned representations encoded by GCNNs may be better at extracting structure-activity relationships from smaller molecules that extrapolate in chemical space to larger molecules.

Splits are schemes designed purely to assess predictive performance. When it comes to building models for deployment to medicinal chemistry teams, all available data are used for training, and, in practice, the predictive performance for lead optimization is typically slightly better than that reported in the studies discussed above. In general, machine learning approaches perform better with multiple experimental observations and molecular diversity. Repeated observations are useful for quantifying experimental variability in the assay and therefore the limits of predictability $[1,17]$. Molecular diversity in the training data allows models to generalize to a wide range of molecular scaffolds (global diversity) as well as learn nuances from smaller functional group perturbations (localized diversity). A continued discussion between modelers and chemists can lead to selection of molecules that fill in model gaps to ultimately improve predictions. We next discuss methods that can help direct such discussions.

\section{Interpretability, error \& the use of deep learning models}

As DNN models increasingly are applied in drug discovery, users will want information on a model's underlying molecular assumptions, uncertainty for individual predictions and chemical domain validity. Deep learning methods currently fall short of regression and recursive partitioning approaches (such as RF and Cubist) in these areas, although addressing these shortcomings are the focus of active, burgeoning work [2,3].

Interpretability is important in allowing scientists to 'sanity check' structure-activity relationships identified by a model and identify spurious correlations $[1,2]$ which restrict rather than improve drug discovery. Current interpretative approaches can be broken down into three types: quantifying prediction changes at a specific heavy atom from in silico addition or deletion of a fragment to the atom position, quantifying the effect of a particular input feature based on a DNN's internal learned weight matrix and building separate explanation models that approximate a complex model that is not interpretable (such as Shapley additive explanations) $[2,3,8,14,18]$. These 
approaches highlight substructures on a molecule that contribute strongly to a model's prediction and enable both human review and further molecular design. We expect this area to develop significantly in the next couple years.

In addition to interpretability, users can benefit from reliable estimates for when an individual prediction is a confident one or is beyond the scope of the model's underlying assumptions. Hirschfeld et al. recently categorized error estimation approaches for deep learning methods as falling into four broad categories: using ensembles of models generated with multiple parameter or training sets, creating DNN models that directly estimate a standard deviation along with a predicted value, calculating uncertainty based on distance from closely related training molecules and combining DNN outputs with methods like RF that have established approaches to estimate uncertainty [19].

It is important to separate the concepts of errors intrinsic to assays themselves (aleatoric error) from errors caused by insufficient data and limitations of machine learning models (epistemic uncertainty) as each would result in a different approach to fix the error (such as alternate assays for aleatoric error or increased sampling of chemical space for epistemic uncertainty). Currently, there is little consensus on the overall optimal approach for estimating uncertainty for DNNs, but methods that append a RF model to a GCNN do appear to have a slight edge and error prediction performance does appear to be task dependent [19]. Emerging DNNs known as fully Bayesian networks [19], whose parameters are treated as distributions, also hold promise. As methodologies mature for error estimation, greater confidence may be applied to certain model predictions and molecular spaces to be treated with concern can be highlighted.

As an additional note, while DNNs are considered state-of-the-art for certain tasks, they require a lot of data and computational resources. For low data regimes, traditional methods such as RF or even regression may perform as well or better than DNN approaches and should not be discarded out-of-hand, especially considering that these models have established techniques for interpretability and error estimation [1]. As the field continues to change, the goal for predictive modelers and drug discovery groups will be to continuously review trade-offs between methods.

\section{Future perspective}

We believe that GCNNs will play a major role in ADMET prediction models for medicinal chemistry and their predictive ability has already surpassed RF and fully connected DNNs for a subset of end points. So where will the next improvements in ADMET predictions come from? We suggest three areas.

The first is increased incorporation of mechanistic understanding and biophysical information that DNNs must currently attempt to infer. The model interpretability tools discussed above provide insights that can be matched to mechanistic understanding [1]. Biophysical information can include quantum chemical properties and 3D properties such as molecular flexibility, conformational energies and biomolecular interactions (ion channels and CYPs, for instance). For more complex biological end points, such as those from in vivo pharmacokinetic studies, incorporating modeling of biological processes may help [1].

The second is creative algorithmic advances to improve generalization and reduce reliance on dataset size. Currently, deep learning models may be limited by the range of the chemical diversity, data availability and bias inherent to an institution's focus. One approach from the vision and language realms called 'pretraining' allow a DNN model trained once using very large datasets to be successful in new domains with very small datasets $[1,2]$. While this approach has so far yielded mixed results for chemistry, it is still early days. It also remains to be seen how efforts by researchers to share and combine data from public and private realms can overcome deficiencies in chemistry coverage [4].

Finally, ADMET prediction models can often be better integrated into the iterative medicinal chemistry designmake-test cycle. We note that in our practice, models considered poorly predictive (e.g., hERG models with time split $\mathrm{R}^{2}$ of $\sim 0.3$ ) are still useful in prioritizing molecules, especially in the context of large numbers of molecules and categorical predictions [20]. Nevertheless, methods for highlighting gaps in prediction model 'knowledge' can facilitate design of molecules to explore deficiencies. DNNs using continuous representations that allow for generative creation of new molecules with desired properties may further contribute to efficient lead evolution [1]. We believe a close coupling of graph convolutional ADMET predictors and medicinal chemistry collaboration will improve the speed and reliability of designing new and improved therapeutics. 


\section{Acknowledgments}

The authors thank JC Alvarez, KV Chuang, MJ Keiser, E Joshi and R Sheridan for helpful comments. We unfortunately had to omit many good references given article format limitations.

\section{Financial \& competing interests disclosure}

EL Cáceres is supported under the National Science Foundation Graduate Research Fellowship Program under grant no. 1650113. The authors are currently or previously employed by Merck \& Co., Inc. The authors have no other relevant affiliations or financial involvement with any organization or entity with a financial interest in or financial conflict with the subject matter or materials discussed in the manuscript apart from those disclosed.

No writing assistance was utilized in the production of this manuscript.

\section{Open access}

This work is licensed under the Attribution-NonCommercial-NoDerivatives 4.0 Unported License. To view a copy of this license, visit http://creativecommons.org/licenses/by-nc-nd/4.0/

\section{References}

1. Schneider P, Walters WP, Plowright AT et al. Rethinking drug design in the artificial intelligence era. Nat. Rev. Drug Discov. 19(5), 353-364 (2020).

2. Chuang K V, Gunsalus LM, Keiser MJ. Learning molecular representations for medicinal chemistry. J. Med. Chem. 63(16), 8705-8722 (2020).

3. Polishchuk P. Interpretation of quantitative structure-activity relationship models: past, present, and future. J. Chem. Inf. Model. 57(11), 2618-2639 (2017).

4. Ma J, Sheridan RP, Liaw A, Dahl GE, Svetnik V. Deep neural nets as a method for quantitative structure-activity relationships. J. Chem. Inf. Model. 55(2), 263-274 (2015).

5. Kearnes S, Goldman B, Pande V. Modeling industrial ADMET data with multitask networks. arXiv:1606.08793 (2016).

6. Zhou Y, Cahya S, Combs SA et al. Exploring tunable hyperparameters for deep neural networks with industrial ADME data sets. J. Chem. Inf. Model. 59(3), 1005-1016 (2019).

7. Montanari F, Kuhnke L, Ter Laak A, Clevert D-A. Modeling physico-chemical ADMET endpoints with multitask graph convolutional networks. Molecules 25(1), 44 (2019).

8. Wenzel J, Matter H, Schmidt F. Predictive multitask deep neural network models for ADME-Tox properties: learning from large data sets. J. Chem. Inf. Model. 59(3), 1253-1268 (2019).

9. Xu Y, Ma J, Liaw A, Sheridan RP, Svetnik V. Demystifying multitask deep neural networks for quantitative structure-activity relationships. J. Chem. Inf. Model. 57(10), 2490-2504 (2017).

10. Duvenaud DK, Maclaurin D, Iparraguirre J et al. Convolutional networks on graphs for learning molecular fingerprints. In: Advances in Neural Information Processing Systems 28. Cortes C, Lawrence ND, Lee DD, Sugiyama M, Garnett R (Eds). Curran Associates, Inc,NY, USA, 2224-2232 (2015).

11. Yang K, Swanson K, Jin W et al. Analyzing learned molecular representations for property prediction. J. Chem. Inf. Model. 59(8), 3370-3388 (2019).

12. Coley CW, Barzilay R, Green WH, Jaakkola TS, Jensen KF. Convolutional embedding of attributed molecular graphs for physical property prediction. J. Chem. Inf. Model. 57(8), 1757-1772 (2017).

13. Feinberg EN, Sur D, Wu Z et al. PotentialNet for molecular property prediction. ACS Cent. Sci. 4(11), 1520-1530 (2018).

14. Feinberg EN, Joshi E, Pande VS, Cheng AC. Improvement in ADMET prediction with multitask deep featurization. J. Med. Chem. 63(16), 8835-8848 (2020).

15. Liu K, Sun X, Jia L et al. Chemi-Net: a molecular graph convolutional network for accurate drug property prediction. Int. J. Mol. Sci. 20(14), (2019).

16. Sheridan RP. Time-split cross-validation as a method for estimating the goodness of prospective prediction. J. Chem. Inf. Model. 53(4), 783-790 (2013).

17. Sheridan RP, Karnachi P, Tudor M et al. Experimental error, kurtosis, activity cliffs, and methodology: what limits the predictivity of quantitative structure-activity relationship models? J. Chem. Inf. Model. 60(4), 1969-1982 (2020).

18. Rodríguez-Pérez R, Bajorath J. Interpretation of compound activity predictions from complex machine learning models using local approximations and shapley values. J. Med. Chem. 63(16), 8761-8777 (2020)

19. Hirschfeld L, Swanson K, Yang K, Barzilay R, Coley C. Uncertainty quantification using neural networks for molecular property prediction. arXiv:2005.10036 (2020).

20. Sanders JM, Beshore DC, Culberson JC et al. Informing the selection of screening hit series with in silico absorption, distribution, metabolism, excretion, and toxicity profiles. J. Med. Chem. 60(16), 6771-6780 (2017). 